\title{
Towards reducing population radiation exposure
}

\author{
Richard Fox
}

Published online: 19 July 2013

(C) Australasian College of Physical Scientists and Engineers in Medicine 2013

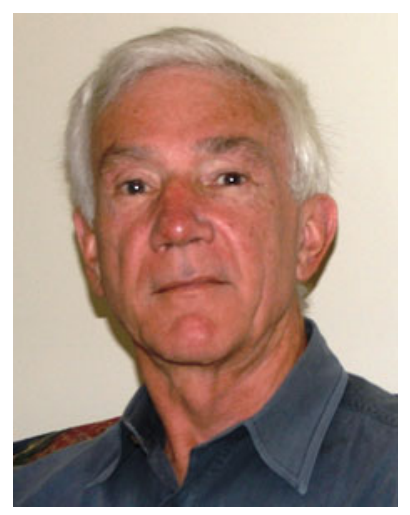

The damaging effect of ionizing radiation has been known about for a very long time, however there has been some dispute about the effect of low doses of radiation typical of diagnostic $\mathrm{x}$-ray and nuclear medicine imaging. Although all international bodies responsible for advice on radiation safety have recommended the assumption that the risk of cancer is linear down to zero dose, there are many who have argued that there is a threshold or even that very low doses are good for you. The National Academy of Science has released the BEIR VII Phase 2 (2006) [1] report which having evaluated 700 references on all aspects of the effects or ionizing radiation, including hormesis, has concluded that "current scientific evidence is consistent with the hypothesis that there is a linear dose-response relationship between exposure to ionizing radiation and the development of radiation-induced solid cancers in

R. Fox $(\bowtie)$

School of Physics, University of Western Australia, Crawley, WA, Australia

e-mail: richard.fox@uwa.edu.au humans". The report also concludes that "it is unlikely that a threshold exists for the induction of cancers".

More recently two papers have been published that directly link the induction of cancer in children with the use of CT scans. In the first of these [2] the authors conclude that compared with children receiving organ doses of $<5 \mathrm{mGy}$, the relative risk of leukaemia after a mean organ dose of $51 \mathrm{mGy}$ was increased by a factor of $3.2(95 \% \mathrm{CI}$ $1.5-7)$ and the relative risk for brain cancer after a mean organ dose of $60 \mathrm{mGy}$ was increased by a factor of 2.8 (95\% CI 1.3-6). The authors conclude that for every 10,000 head CT scans in children there is one extra case of leukaemia and one extra case of brain tumour. The second paper recently published by an Australian group [3] who looked at 680,000 CT scans on children, has shown a very strong correlation between performing a scan and the subsequent induction of cancer. This latter paper estimates that a single CT scan in children (60\% of which were head scans) increases the risk of cancer by $\sim 16 \%$. Of course, it needs to be kept in mind that this still represents an extremely small risk, since the natural incidence is so low. However, a risk certainly appears to exist and it is important that we limit this to the lowest value possible.

Apart from the two recent papers, most of the evidence for the induction of cancer at low doses comes from the analysis of the 'atomic bomb' data. This data is very persuasive at high doses but is substantially less accurate and therefore less convincing at the type of doses delivered in diagnostic radiology and nuclear medicine. Nevertheless, there is evidence $[4,5]$ that doses of the order of $10 \mathrm{~s}$ of $\mathrm{mSv}$ do increase the risk of cancer, although the certainty of this data is less than the $95 \%$ confidence normally demanded when quoting scientific results. However when dealing with patient safety it behoves the medical profession to be conservative. If the need for a conservative 
approach associated with the risk of ionizing radiation conflicted with the need for appropriate diagnosis of patient illness, one would have to make a choice between the two. However the risk of radiation induced cancer from a diagnostic test is extremely low and has a latent period of approximately 10 years. This means that in almost every situation, IF A PATIENT WILL BENEFIT FROM A DIAGNOSTIC TEST THAT USES IONIZING RADIATION, THEN THEY SHOULD HAVE THE TEST, because the benefit will almost certainly outweigh the risk. Consequently we should be addressing our attention to the reduction in the unnecessary use of ionizing radiation. That is, the use of imaging tests that are not required either because imaging will not provide useful information and will not change diagnosis or treatment or because a different test using less or no ionizing radiation is preferred. It has been estimated in several papers [6-8] that more than $30 \%$ of imaging tests are inappropriate and should not have been requested. Thus there is considerable scope for dose reduction and therefore improvement in patient care.

Physicists and engineers working in radiology should be actively promoting the appropriate use of ionizing radiation and doing what we can to discourage its inappropriate use. The use of ionizing radiation tests that are not required for the patient not only irradiates patients unnecessarily, but adds cost to the health system and delays diagnosis, since if the wrong test is requested then the correct test is not being done. In addition, since all tests have false positives, the application of an inappropriate test will lead to a significant number of patients being further investigated for no purpose, with consequent risk, cost and disruption. Hence it is in everyone's interest to reduce unnecessary tests as much as possible. The main target for this is the GP because they are subject to a number of pressures that lead to unnecessary patient irradiation. In the first place there is pressure from patients themselves who will sometimes come to their doctor demanding a particular test. In addition, the imaging procedures available are continually changing and which test is the most appropriate in any particular circumstance is even difficult for radiologists and nuclear medicine physicians to keep up with. Finally GPs are under significant threat of litigation if a patient develops a condition that would have been detected earlier by a diagnostic test that had not been requested by the GP.

To assist in reducing inappropriate imaging a website has been developed by a team at Royal Perth Hospital [9] that informs doctors of the appropriate diagnostic imaging test when the patient symptoms are known. This site utilises the World Wide Web and is freely available at 'www.imagingpathways.health.wa.gov.au'. It includes common clinical scenarios and has a flow chart structure which focuses on the symptoms rather than the pathology. Its layered format means that summary information is provided that can be investigated in more detail if the user requires. The difficulty is to ensure that GPs and specialists requesting $\mathrm{X}$-ray and nuclear medicine tests are familiar with this web site and make use of it when required. To help achieve the aim of reducing unnecessary imaging, a number of steps have been taken in Western Australia. All medical graduates from the University of Western Australia are now required to pass a module in radiation safety which includes information about the risk of ionizing radiation and incorporates questions that require the use of the diagnostic imaging pathways web site. This module and its associated quiz are freely available on the Diagnostic Imaging Pathways web site. Agreement has also been reached with the Dean of the Medical School at Notre Dame University that he will introduce a similar requirement for their medical students. In addition, the Western Australian Health Department's Medical Directors Forum has determined that all new clinical appointments to Health Department Hospitals will be required to pass this radiation module. This will mean that within a few years a significant number of doctors in Western Australia will have had exposure to the imaging pathways web site and will have some understanding of the potential risks of ionizing radiation. Recently ARPANSA has shown interest in expanding the use of the radiation module throughout Australia and discussions are proceeding as to how this might be implemented.

In addition to reducing the unnecessary use of ionizing radiation, it is also important to ensure that the radiation dose used in imaging tests is as low as possible consistent with diagnostic quality images. This is something in which members of the ACPSEM (the College) should be playing an active role. It is well known that there is a significant discrepancy in the radiation dose delivered between different radiology services for apparently the same investigation. Several years ago a paper that considered CT doses in Australia [10] showed that the ratio between the highest and lowest doses for apparently the same procedure varied by up to a factor of 36. Although there are valid reasons why different services may deliver different doses, it seems likely that much of this factor of 36 represents overdosing by some radiology services. The CT industry has in recent years introduced a number of dose limiting initiatives the most recent and significant being the introduction of iterative reconstruction. In principle this can substantially reduce patient dose, however it will only do so if other factors are adjusted so that the noise level is restored to the level obtained with back projection reconstruction. Consequently image optimisation $[11,12]$ is a crucial step in ensuring that patient doses are as low as reasonably achievable.

ARPANSA has recently established dose reference levels (DRL) [13] for CT in Australia. By advising 
radiologists how their doses compare with others in Australia it is hoped that some of the services delivering higher doses will reduce their doses to make them more comparable with services that deliver a lower dose. This initiative has been actively promoted and supported by members of the College.

Thus College members can play a significant role in the reduction of patient dose. In the first place by encouraging mechanisms that ensure that all diagnostic procedures using ionizing radiation are only performed for the benefit of the patient and secondly by helping to ensure that diagnostic procedures use the minimum dose consistent with adequate diagnostic imaging quality. These are aims that all College members working in relevant fields should be actively addressing.

Acknowledgments I would like to acknowledge support and helpful comments on the manuscript from Clinical Professor Richard Mendelson of Royal Perth Hospital.

\section{References}

1. Monson RR, Cleaver JE, Abrams HL, Bingham E, Buffler PA et al (2006) Health Risks from exposure to low levels of ionizing radiation BEIR VII Phase 2 The National Academies Press (www.nap.edu/catalog/11340.html)

2. Pearce MS, Salotti JA, Little MP, McHugh K, Lee C, Kim KP et al (2012) Radiation exposure from CT scans in childhood and subsequent risks of leukaemia and brain tumours: a retrospective cohort study. Lancet 380(9840):499-505

3. Mathews JD, Forsythe AV, Brady Z, Butler MW, Goergen SK, Byrnes GB et al (2013) Cancer risk in 680,000 people exposed to computed tomography scans in childhood or adolescence: data linkage study of 11 million Australians. BMJ 346:f2360

4. Pierce DA, Shimuzu Y, Preston DL, Vaeth M, Mabuchi K (1996) Studies of mortality of atomic bomb survivors Report 12, Part 1. Cancer: 1950-1990. Rad Res 146(1):1-27

5. Pierce DA, Preston DL (2000) Radiation-related cancer risks at low doses among atomic bomb survivors. Rad Res 154:178-186

6. Bairstow PJ, Persaud J, Mendelson R, Long N (2010) Reducing inappropriate diagnostic practice through education and decision support. Int J Qual Health Care 22(3):194-200

7. Simpson G, Hartrick G (2007) Use of thoracic CT by general practitioners. Med J Aus 187:43-46

8. Lehnert BE, Bree RL (2010) Analysis of the appropriateness of outpatient CT and MRI referred from primary care clinics at an academic medical center: how critical is the need for improved decision support? J Amer Coll Radiol 7(3):192-197

9. Mendelson R, Bairstow PJ (2009) Imaging pathways: will they be well trodden or less travelled? J Am Coll Radiol 6(3):160-166

10. Moss M, McLean D (2006) Paediatric and adult computed tomography practice and patient dose in Australia. Aus Radiol 50(1):33-40

11. Heggie JCP (2005) Patient doses in multi-slice CT and the importance of image optimisation. Australas Phys Eng Sci Med 28(2):86-96

12. Heggie JCP, Kay JK, Lee WK (2006) Importance in optimization of multi-slice computed tomography scan protocols. Aus Radiol 50(3):278-285

13. Hayton A, Wallace A, Marks P, Edmonds K, Tingey D, Johnston $P$ (2013) Australian diagnostic reference levels for multi-detector computed tomography. Aust Phys Eng Sci Med 36(1):19-26 